\title{
The perfect crime? CCSVI not leaving a trace in MS
}

\author{
Christoph A Mayer, ${ }^{1}$ Waltraud Pfeilschifter, ${ }^{1}$ Matthias W Lorenz, ${ }^{1}$ Max Nedelmann, ${ }^{2}$ \\ Ingo Bechmann, ${ }^{3}$ Helmuth Steinmetz, ${ }^{1}$ Ulf Ziemann ${ }^{1}$
}

\section{See Editorial Commentary,} p 355

- Additional tables are published online only. To view these files please visit the journal online (http://jnnp.bmj. com)

'Department of Neurology, Goethe-University Frankfurt, Frankfurt am Main, Germany ${ }^{2}$ Department of Neurology, Justus-Liebig-University Giessen, Giessen, Germany ${ }^{3}$ Institute of Anatomy, University of Leipzig, Leipzig, Germany

\section{Correspondence to} Dr Christoph A Mayer, Department of Neurology, Goethe-University Frankfurt, Schleusenweg 2-16, 60528 Frankfurt am Main, Germany; christoph.mayer@kgu.de

CAM and WP contributed equally.

Received 8 October 2010 Revised 8 December 2010 Accepted 11 December 2010 Published Online First 4 February 2011

\begin{abstract}
Background Multiple sclerosis (MS) is a chronic, inflammatory demyelinating disease of the central nervous system, believed to be triggered by an autoimmune reaction to myelin. Recently, a fundamentally different pathomechanism termed 'chronic cerebrospinal venous insufficiency' (CCSVI) was proposed, provoking significant attention in the media and scientific community.
\end{abstract}

Methods Twenty MS patients (mean age 42.2 \pm 13.3 years; median Extended Disability Status Scale 3.0, range $0-6.5$ ) were compared with 20 healthy controls. Extra- and intracranial venous flow direction was assessed by colour-coded duplex sonography, and extracranial venous cross-sectional area (VCSA) of the internal jugular and vertebral veins (IJV/VV) was measured in B-mode to assess the five previously proposed CCSVI criteria. IJV-VCSA $\leq 0.3 \mathrm{~cm}^{2}$ indicated 'stenosis,' and IJV-VCSA decrease from supine to upright position 'reverted postural control.' The sonographer, data analyser and statistician were blinded to the patient/control status of the participants.

Results No participant showed retrograde flow of cervical or intracranial veins. IJV-VCSA $\leq 0.3 \mathrm{~cm}^{2}$ was found in $13 \mathrm{MS}$ patients versus 16 controls $(p=0.48)$. A decrease in IJV-VCSA from supine to upright position was observed in all participants, but this denotes a physiological finding. No MS patient and one control had undetectable IJV flow despite deep inspiration $(p=0.49)$. Only one healthy control and no MS patients fulfilled at least two criteria for CCSVI.

Conclusions This triple-blinded extra- and transcranial duplex sonographic assessment of cervical and cerebral veins does not provide supportive evidence for the presence of CCSVI in MS patients. The findings cast serious doubt on the concept of CCSVI in MS.

\section{INTRODUCTION}

Multiple sclerosis (MS) is a chronic, inflammatory disease of the central nervous system, with substantially heterogeneous clinical features due to variable extents of degeneration and inflammation. The prevalence is estimated at 30/100000, translating to more than two million MS-patients worldwide. The hallmark of MS is the perivenous white-matter lesion, ${ }^{1}$ characterised by local inflammation, de- and remyelination, and local astrogliosis. ${ }^{2}$ The most widely accepted hypothesis on the origin of $\mathrm{MS}$ is a primary autoimmune disease, mediated by auto-reactive, oligodendrocyte-specific T cells. ${ }^{3} 4$

Recently a new hypothesis on the genesis of MS was raised, fundamentally divergent from established autoimmunological theories. ${ }^{5-7}$ In a recent study ${ }^{5}$ based on duplex sonographic and venographic assessment of extracranial and intracranial veins of $65 \mathrm{MS}$ patients and 235 controls, Zamboni et al claimed a perfect coincidence of MS and venous stenoses in various locations. Proposing a pathophysiological mechanism of 'venous congestion,' analogous to lower-limb chronic venous insufficiency (CVI), ${ }^{8}$ they interpreted the predominantly venotopic location of MS lesions as a consequence of local erythrocyte extravasation owing to elevated transmural venous pressure, followed by erythrocyte degradation and iron-driven phagocytosis. ${ }^{6}$ This concept was named 'chronic cerebrospinal venous insufficiency' (CCSVI). 56

The idea of venous congestion as a possible contributor to the pathogenesis of MS has been discussed for the past 40 years, ${ }^{9-12}$ but remained widely unappreciated by the scientific community. Until recently, no scientific attempt was undertaken to prove or disprove the hypothesis despite its potential therapeutic implications. Therefore, the exceptionally significant results presented by Zamboni et al ${ }^{5}{ }^{6}$ generated extraordinary interest. Is it possible that we have overlooked something so obvious for so long?

The aim of this study was to confirm the data presented by Zamboni et al. ${ }^{5} \mathrm{~A}$ critical and independent re-evaluation of the CCSVI hypothesis is of particular importance, given that attempts of endovascular treatment by percutaneous transluminal angioplasty, as suggested by Zamboni and coworkers,' were already complicated by serious adverse events in two cases. ${ }^{13}$

\section{METHODS}

\section{Subjects}

Twenty MS patients and 20 healthy controls participated (table 1). Exclusion criteria for patients and controls were: previous history of thrombophilia or cerebrovascular diseases including migraine, idiopathic intracranial hypertension, intracranial sinus thrombosis, thrombosis of jugular veins and neck dissection. Patients with a relapse within 30 days before investigation were excluded. All participants gave their written informed consent. We included 17 relapsing-remitting and three secondary-progressive patients from our MS outpatient clinic. All patients fulfilled the revised McDonald diagnostic criteria. ${ }^{15}$ This study was approved by the ethics committee of the Faculty of Medicine, Goethe-University Frankfurt.

\section{Ultrasound examination}

Investigations were performed by a trained expert sonographer (WP), who was blinded to the MS-patient/healthy control attribution of each participant. This was achieved by transferring the 
Table 1 Clinical and demographic data of multiple sclerosis (MS) patients and healthy control subjects

\begin{tabular}{|c|c|c|c|c|}
\hline Demographics & Relapsing-remitting MS & Secondary progressive MS & All MS & Controls \\
\hline Subjects, no & 17 & 3 & 20 & 20 \\
\hline Mean age, years $(S D)$ & $40.3(13.9)$ & $55.3(9.3)$ & $42.2(13.3)$ & $34.3(11.0)$ \\
\hline Sex male/female & $5 / 12$ & $2 / 1$ & $7 / 13$ & $10 / 10$ \\
\hline Disease duration, years, range/mean/SD & $1-35 / 11.2 / 10.2$ & $11-36 / 23 / 12.5$ & $1-36 / 13.1 / 11.1$ & / \\
\hline Patients on medication, no & $15 / 17$ & $3 / 3$ & $18 / 20$ & / \\
\hline CSF positive $\nmid$, no/total & $16 / 17$ & $3 / 3$ & $19 / 20$ & / \\
\hline MRI positive $\ddagger$, no/total & $17 / 17$ & $3 / 3$ & $20 / 20$ & / \\
\hline
\end{tabular}

*In the previous 12 months.

†Positive oligoclonal bands.

$\neq$ Cerebral MRI indicative for MS according to Barkhof et al. ${ }^{14}$

participants to the laboratory by a nurse, comfortably positioning them on a tilt chair and covering them with a blanket to cover any hints such as injection marks potentially allowing for group assignment. Participants were instructed not to talk to prevent clues by possible dysarthria. The sonographer did not enter the laboratory until these precautions were taken. A Philips IU22 (Böblingen, Germany) ultrasound system with an L9-3 probe was used for assessment of the IJV/VV, and transcranial measurements were performed with an S5-1 probe (both Philips). Special attention was paid when scanning the IJV not to exert pressure on the vessel. The investigation was carried out following the five criteria suggested by Zamboni et al :

1. Reflux in the internal jugular veins (IJVs) and/or vertebral veins (VVs) in the supine and sitting position. Flow characteristics of both IJVs and VVs were investigated in $0^{\circ}$ supine followed by $90^{\circ}$ upright sitting positions. Reflux in any vein $>0.88 \mathrm{~s}$ was considered 'pathological. ${ }^{5}$ Data were recorded during a period of $3-5 \mathrm{~s}$ of apnoea following normal exhalation. The probe was located in a longitudinal plane between vertebrae C6 and C7, which was maintained when participants changed to upright position.

2. Reflux in the deep cerebral veins (DCVs). The Vv. basales Rosenthal, -cerebri media profunda (VCP) and -Galeni (VG) were identified according to standards described elsewhere. ${ }^{16}$ In addition, the sinus transversus was assessed. Flow characteristics in at least one DCV or sinus transversus were measured. Flow in a reversed direction $>0.5 \mathrm{~s}$ was considered 'pathological.'

3. High-resolution B-mode evidence of IJV stenoses. The narrowest venous cross-sectional area (VCSA) in the accessible parts of the IJV was measured in a transversal plane in supine position. VCSA $\leq 0.3 \mathrm{~cm}^{2}$ indicated 'stenosis.'

4. Flow not Doppler-detectable in the IJVs and/or VVs. Flow in both IJV and VV was assessed in supine and upright positions. Lack of Doppler-detectable flow following deep inspiration despite a detectable lumen was considered 'pathological. ${ }^{5}$

5. Reverted postural control of the main cerebral venous outflow route measured in IJV. $\triangle$ VCSA $_{\text {upright-supine of the IJV was }}$ quantified. A positive value indicated 'loss of postural control of the predominant outflow route in the supine position. ${ }^{5}$ The rationale for this modification of the respective "Zamboni-criterion" is explained in table 2 and the Results.

\section{Offline and statistical analyses}

The sonographer performed no data analysis but stored sonographic data as single frames (criteria 3 and 5) or 'cine' files (criteria 1,2 and 4). Stored data were evaluated offline by a second investigator ('rater') who was blinded to all demographics (including age and gender). Statistical analyses were carried out on partially unmasked data. The statistician (MWL) was only aware which subjects belonged to 'group A' or 'group B' without knowing which of these groups were patients or controls. Given all blinding procedures, investigations and analyses were 'tripleblind.' Intergroup comparisons were carried out with the Student t test after confirmation of normality with QQ plots. Categorial variables were compared with the Fisher exact test (dichotomous variables) or the $\chi^{2}$ homogeneity test (more than two categories). A p value of $<0.05$ was regarded as statistically significant, and all tests were carried out two-tailed. Data are given as means \pm SD if not otherwise indicated. All analyses were carried out with the SAS 9.1 software package.

\section{RESULTS}

Two patients and one healthy control had no transtemporal ultrasound window. IJVs and VVs were detectable in $95 \%$ of participants in the supine and in $97.5 \%$ in the upright position. Detection rates of at least one DCV reached $92.5 \%$ in the supine and $87.5 \%$ in the upright position, thus showing a good match with the rates reported elsewhere. ${ }^{16}$ There was no significant difference in detection rates between MS patients and healthy controls. Detailed statistics are given in supplementary tables 1, 2 .

\section{Reflux in the IJVs and/or VVs in supine and sitting position}

Flows in IJVs and VVs were strictly orthograde and unidirectional, regardless of position and group. Reflux $>0.88 \mathrm{~s}$ was not seen in any participant.

\section{Reflux in the DCVs}

In all 18 MS-patients and 19 controls with detectable DCVs, flow was strictly orthograde and unidirectional, regardless of position. Reflux $>0.5 \mathrm{~s}$ was not seen in any participant.

\section{High-resolution B-mode evidence of IJV stenoses}

VCSA $\leq 0.3 \mathrm{~cm}^{2}$ was found in the right IJV of $20 / 40(50 \%)$ participants, in the left IJV of $27 / 40(67.5 \%)$ participants and bilaterally in $18 / 40$ (45\%) participants. The combined incidence was $29 / 40(72.5 \%)$ participants. The minimum VCSA was $0.32 \pm 0.15 \mathrm{~cm}^{2}$ in the right IJV (range $0.05-0.81 \mathrm{~cm}^{2}$ ) and $0.26 \pm 0.17 \mathrm{~cm}^{2}$ in the left IJV (range $0.03-0.72 \mathrm{~cm}^{2}$ ). There was no significant group difference in the incidence of single or combined ICV-VCSA $\leq 0.3 \mathrm{~cm}^{2}$ (13 MS patients $(65 \%)$ vs 16 controls $(80 \%), p=0.48)$. The minimal right IJV-VCSA was not different between MS patients and controls $\left(0.31 \pm 0.13 \mathrm{~cm}^{2}\right.$ vs $\left.0.33 \pm 0.19 \mathrm{~cm}^{2}, \mathrm{p}=0.65\right)$, while the minimal left IJV-VCSA was significantly larger in MS patients than controls $\left(0.33 \pm 0.18 \mathrm{~cm}^{2}\right.$ vs $0.19 \pm 0.12 \mathrm{~cm}^{2}, \mathrm{p}=0.005$ ) 
Table 2 Fulfillment of the five 'Zamboni criteria' in $20 \mathrm{MS}$ patients and 20 healthy controls

\begin{tabular}{|c|c|c|c|c|}
\hline Criterion & Definition according to 'Zamboni protocol'* & No of MS patients & No of controls & p Value $\dagger$ \\
\hline 1 & Reflux $>0.88 \mathrm{~s}$ in the IJVs and/or VVs in sitting or supine position & 0 & 0 & / \\
\hline 2 & Reflux $>0.5 \mathrm{~s}$ in the deep cerebral veins & 0 & 0 & / \\
\hline 3 & High resolution B-mode evidence of IJV stenoses defined as VCSA of $\leq 0.3 \mathrm{~cm} \dagger \neq$ & 13 & 16 & 0.48 \\
\hline 4 & Flow not Doppler-detectable in at least one IJV or VV§ in the supine and upright position & 0 & $1 \S$ & 1.0 \\
\hline 5 & Atypical main cerebral venous outflow measured in IJV defined as $\Delta V C^{\prime} S A_{\text {upright-supine }}>0$ I & 0 & 0 & I \\
\hline \multicolumn{2}{|c|}{ At least two criteria fulfilled } & 0 & 1 & 1.0 \\
\hline
\end{tabular}

*Zamboni et al. ${ }^{5}$

†Fisher exact test.

$\neq$ Criterion 3 was not defined consistently by Zamboni et al. We used the definition in Zamboni et $a l_{1}^{5}$ where a venous cross-sectional area (VCSA) of $\leq 0.3 \mathrm{~cm}{ }^{2}$ is given as a cut-off value. Doepp et $a^{21}$ obtained different results in assessing criterion 3, but referred to a deviating definition of 'stenosis' given in Zamboni et al, ${ }^{25 a} \mathrm{~J}$ Neurol Sci 2009;282:21-7, where a local VCSA reduction of $\geq 50 \%$ is considered 'stenotic.'

$\S$ Criterion 4 is not specified clearly by Zamboni et al. Here, we defined criterion 4 as 'no flow detectable in at least one internal jugular vein (IJV) or vertebral vein (VV) in the supine and the upright position.'

IThis Zamboni criterion was modified. Given that negative values for $\Delta$ VCSA in the IJV represent not a pathological but a physiological state, this criterion was substituted by 'atypical flow, /, indicates $\mathrm{p}$ value could not be calculated. indicating a positive value of $\triangle \mathrm{VCSA}$.

A moderate positive correlation between age and VCSA was found (right IJV: $r=0.39, p=0.014$; left IJV: $r=0.55, p<0.001$ ). To exclude potential bias by age interaction in the blinded analysis, a secondary analysis of a subset of 24 subjects (12 MS patients, 12 controls) was performed, where MS patients and controls were randomly drawn matched by age (MS patients: $34.4 \pm 10.6$ years; controls: $34.9 \pm 11.6$ years, $p=0.91)$. In these age-matched subpopulations, significant group differences in the minimal VCSA of the right or left IJV were no longer present.

\section{Flow not Doppler-detectable in the IJVs and/or VVs}

In two controls but no MS patients $(p=0.49)$, venous flow was absent $(1 \times$ supine, $1 \times$ supine + upright position, left VV) despite a detectable lumen.

\section{Reverted postural control of the main cerebral venous outflow route measured in IJV}

A negative value of $\Delta \mathrm{VCSA}_{\text {upright-supine }}$ was found in the IJVs of all participants. However, this does not indicate 'loss of postural control of the predominant outflow route in the supine position' as implicated in the 'Zamboni protocol' but constitutes the typical physiological response in healthy subjects. ${ }^{17-20}$ Therefore, a negative value of $\Delta V C S A_{\text {upright-supine }}$ in the IJVs was considered normal, and the Zamboni criterion was changed to 'atypical flow,' indicating a positive value of $\Delta \mathrm{VCSA}_{\text {upright-supine }}$ (see table 2).

Altogether, only one healthy control fulfilled $\geq 2$ criteria for CCSVI. The results are summarised in table 2 .

\section{DISCUSSION}

The CCSVI ${ }^{6}$ hypothesis has attracted significant attention in the media and scientific community. Endovascular treatment by percutaneous transluminal angioplasty, as suggested by Zamboni and coworkers, ${ }^{7}$ was, however, complicated by serious adverse events in two cases. ${ }^{13}$ The credibility of the CCSVI concept has already been questioned by two recent studies which could not confirm the findings by Zamboni et al when using extra- and transcranial colour-coded sonography ${ }^{21}$ or phase-contrast magnetic resonance imaging and contrastenhanced magnetic resonance angiography. ${ }^{22}$ Here we sought to replicate the data recently presented by Zamboni et al ${ }^{5}$ by using exactly the same sonography protocol, but for the first time in a triple-blinded controlled study design.

Zamboni and coworkers investigated 65 MS patients and 235 healthy controls in a two-step procedure. ${ }^{5}$ First, screening was performed according to the 'protocol' described above. In case of two or more criteria being fulfilled, angiography was applied. ${ }^{5}$ Because angiography was conducted unblinded, the validity of the screening procedure is crucial for the validity of the whole study.

The first criterion of the 'Zamboni protocol' claims to assess extracranial venous 'reflux' in the IJV and VV by applying a threshold of $0.88 \mathrm{~s}$ to discriminate physiological reversal flow due to valve closure from longer-lasting reflux, assumed to indicate CCSVI. Thus, defined reflux was never seen in our participants, regardless of group. The validity of this threshold value is, however, questionable. It stems from a study on IJV valve insufficiency, ${ }^{23}$ where retrograde flow jets through insufficient valves were found to last $>1.23 \mathrm{~s}$, while physiological backward flows during normal valve closure lasted $0.22-0.78 \mathrm{~s}$. In this context, a threshold of $0.88 \mathrm{~s}$ allows for discrimination between physiological reflux during valve closure and retrograde insufficiency flow. This was, however, assessed during a controlled Valsalva manoeuvre (VM), constituting an entirely different physiological condition compared with when no VM is applied, as in the Zamboni study. ${ }^{5}$ The rationale for transferring this threshold, initially established to assess venous valve insufficiency to the unrelated context of CCSVI where it will serve to assess reflux without association to valve insufficiency, remains unclear and is not scientifically sound; even more so as conditions of measurement were different by omitting VM. Furthermore, the 'Zamboni protocol' does not require assessment of IJV valves. This is another major shortcoming because IJV insufficiency is directly linked to the function of jugular valves. Also, the incidence of jugular valve insufficiency can be as high as $29 \%$ in the normal population, ${ }^{23}$ whereas the incidence of $\mathrm{MS}$ is comparatively very low, approximating $0.03 \%$. Therefore, reflux in the IJV is 1000 times more likely to indicate insufficiency of IJV valves than MS, unless excluded by an experienced sonographer. While jugular valve insufficiency was shown to be linked to certain neurological disease entities, such as transient global amnesia and idiopathic intracranial hypertension, ${ }^{24} 25$ its direct pathological significance has not yet been established. It may likely be viewed as a non-pathological phenomenon in a relevant proportion of humans.

Second, Zamboni et al applied a threshold of $0.5 \mathrm{~s}$ to discriminate pathological 'reflux' in the DCVs, which they found in $54 \%$ of MS patients. ${ }^{5}$ In contrast, we did not detect intracranial venous reflux in any participant. Transcranial assessment of flow direction in DCVs is, however, ambitious because mainly short venous sections with weak duplex signals are detectable. This may easily lead to misinterpretation of flow direction and false-positive results. Furthermore, Zamboni et al derived the 
threshold of $>0.5 \mathrm{~s}$ from phlebological studies in CVI, ${ }^{26}{ }^{27}$ where it serves to quantify venous valve insufficiency following deflation of a tourniquet. The rationale for adopting this value, validated exclusively for assessment of the posterior tibial vein, ${ }^{28}$ to perform a DCV assessment in CCSVI is unclear. Under the likely assumption of unequal hydrostatic and hydrodynamic conditions present in the veins of the leg and those of the brain, failure to validate a threshold criterion for DCV reflux constitutes an unjustified omission that casts doubt on the validity of any data, based hereupon. Unless a clinical syndrome is verifiably directly linked to chronic venous congestion, we strongly recommend avoidance of the expression CVI in this context of cerebral venous drainage.

Third, the 'Zamboni protocol' requires assessment of IJV-VCSA, applying a cut-off value of $\leq 0.3 \mathrm{~cm}^{2}$ that was 'never measured in normal subjects' but reported in $37 \%$ of $M S$ patients. ${ }^{5}$ We found an even higher incidence of low IJV-VCSA (65\% in MS patients, $80 \%$ in controls). Therefore, IJV-VCSA $\leq 0.3 \mathrm{~cm}^{2}$ seems unrelated to $M S$ but seems to represent a common finding also in healthy adults. Furthermore, the cut-off applied was taken from a study on a cohort of patients on an intensive care unit. ${ }^{29}$ Those authors found VCSAs $\leq 0.4 \mathrm{~cm}^{2}$ in $23 \%$ of 160 IJVs, but because of hypovolaemia, mechanical ventilation and other confounds, these data are likely unsuitable to serve as a normative reference for assessment of IJV stenoses. Another limitation in assessing 'stenoses' solely based on IJV-VCSA is its low specificity because, owing to thin vessel walls, even mild pressure exerted by the ultrasound probe inevitably alters the vein diameter, likely leading to false-positive results. By measuring venous blood volume flow, which is independent of these problems, one recent study conducted by Doepp et al found no difference between MS patients and healthy controls. ${ }^{21}$ These considerations raise serious doubts as to whether the criterion VCSA of $\leq 0.3 \mathrm{~cm}^{2}$ can be considered valid with respect to what it aims to measure.

Doepp et al found different results from ours. They did not detect any venous stenoses in their participants. This incongruence is likely explained by differences in the applied definitions of 'stenosis' (see table 2).

Fourth, 'lack of flow' in IJV and/or VV despite deep inspiration was considered to provide indirect evidence for stenosis, and an incidence of $52 \%$ in MS patients but only $3 \%$ in the control group was reported. ${ }^{5}$ In contrast, this abnormality was not observed in any MS patients in the present study. Limitations arise from an unclear definition of this criterion (see table 2) but also from the lack of its validation, which was derived from a study addressing extrajugular venous drainage pathways. ${ }^{30}$ Those authors found evidence for compensational flow increases in the vertebral plexus, while in $3 / 50$ subjects, a jugular flow of $<30 \%$ of global arterial blood flow was seen despite a detectable lumen; this finding, however, was never discussed in the context of 'stenosis.' Moreover, flow assessments were performed at rest rather than during deep inspiration, which constitutes a different physiological state. ${ }^{20}$

The fifth screening parameter, a negative value of IJV-

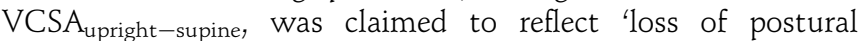
control of the predominant outflow route in the supine position' and reported in $55 \%$ of the MS patients. ${ }^{5}$ All of the participants of our study showed negative VCSA values. Negative $\triangle$ VCSA, however, reflects normal conditions in healthy subjects, as predominant outflow via IJV in the supine but via VV and deep cerebral veins in the upright position is typically found. ${ }^{31}$ Moreover, careful revisitation of the studies cited by Zamboni et $a l^{5}$ reveals that a negative $\triangle$ VCSA reflects indeed the physi- ological cerebral venous drainage in healthy subjects. ${ }^{17-20}$ Zamboni et $a l^{5}$ can be interpreted only by assuming that they accidentally confused $\triangle$ VCSA $>0$ (pathological) with $\triangle \mathrm{VCSA}<0$ (physiological).

Ultrasound-based investigation of vessels is known to be susceptible to rater-bias, which becomes an increasingly important issue, when venous signals with an unfavourable signal-to-noise ratio are assessed. Therefore, blinding of sonographer and rater as to the attribution of participants to the patient versus control group within a study setting is crucial. The precautions applied by Zamboni and coworkers, however, remained undefined, and hence the question arises, as to how reliably blinding was accomplished. In our study, sophisticated blinding procedures were applied to ensure the highest possible standards. In doing so, we found no evidence for 'venous congestion' in MS, despite an adoption of the 'Zamboni protocol' that was otherwise as accurate as possible. ${ }^{5}$

After the ultrasound assessment was found to be positive in all $65 \mathrm{MS}$ patients enrolled in their study, Zamboni and coworkers performed and rated venography in an unblinded manner, revealing 'stenoses' of the major venous conductors in variable locations in each patient but none of the 48 controls. ${ }^{5}$ This perfect association of $100 \%$ constitutes a very uncommon finding in biological systems, and therefore careful interpretation of those data is warranted.

The hypothesis of CCSVI raises further questions concerning conceptual plausibility. In correlating four distinct topographical patterns of 'venous obstruction' detected in MS-patients with clinical course, Zamboni et al stated a significant correlation $(p<0.0001)$ between the pattern of bilateral IJV stenosis with both RRMS (44\%) and SPMS (56\%) ${ }^{5}$ which suggested that bilateral IJV stenoses predispose for the development of MS. Evidence for higher incidences of $\mathrm{MS}$ in patients after bilateral neck dissection, which would match complete bilateral IJV occlusion, is, however, lacking. In contrast, venous flow assessments suggested the coexistence of efficient extrajugular drainage pathways instead of showing signs of congestion. ${ }^{32}$ Convincing evidence exists for the paramount relevance of dynamic extrajugular pathways, sufficiently taking over drainage in case of jugular flow reduction. ${ }^{20}$ The capability of the intraspinal and extraspinal vertebral venous system, buffering up to $1000 \mathrm{ml}$, ${ }^{33}$ is attributed to the valveless and freely communicating architecture of vertebral venous networks as well as the presumably huge cumulative VCSA, most likely surpassing and easily substituting that of both IJV. ${ }^{34} 35$ Accordingly, a significant threefold increase in volume flow of those veins was demonstrated following bilateral IJV obstruction. ${ }^{20}$ These data also indicate that venous cerebral drainage cannot be accurately assessed by measurements limited to the IJVs and VVs, but will require additional assessment of the capacity of substitute drainage pathways such as deep cervical veins, epidural and paravertebral plexus.

Within the pathophysiological concept of CCSVI, ${ }^{6}$ significantly elevated transmural pressure constitutes a mandatory requirement. Elevated CSF pressure, analogous to the concept of venous congestion in idiopathic intracranial hypertension, should therefore be a regular consequence of obstructed cerebral venous drainage in CCSVI. ${ }^{36}$ Zamboni et al did not obtain such data from their patients. However, other studies showed normal CSF pressure after bilateral neck dissection unless further obstructing deep cervical drainage pathways by manual pressure. ${ }^{37}$ These findings stress the functional relevance of deep cervical components for cerebral venous drainage in case of complete bilateral IJV obliteration and are clearly non-confirmative for the proposed pathophysiology of CCSVI. In turn, raised intracranial pressure in $\mathrm{MS}$ patients is only rarely 
reported, for example as a consequence of vasogenic oedema caused by extensive space-occupying inflammatory lesions. ${ }^{38}$

Notwithstanding the clear differences from the well-known pathophysiology of CVI of the lower limbs, Zamboni suggested local extravasation of erythrocytes, driven by elevated transmural venous pressure, followed by degradation and consecutive forming of perivenous iron deposits as the core pathophysiological mechanisms of CCSVI. ${ }^{6}$ Perivascular iron is claimed to be a potent chemoattractant, so that local formation of MS lesions is interpreted as a side effect of iron phagocytosis. While leucocytes can be targeted through vascular walls upon induction of a well-characterised apparatus involving adhesion molecules and ectoenzymes, erythrocytes lack all of the respective proteins. The concept of pressure-induced extravasation of erythrocytes through veins ignores basic features of the architecture of brain vessels and the brain-blood barrier. Most importantly, veins and venules, by definition, possess a vascular wall and a tight endothelium, which certainly is non-permissive for cells. ${ }^{39}$ Furthermore, one recent investigation showed normal CSF ferritin levels in MS patients which serves as another piece of evidence against an aetiological role for CCSVI-related parenchymal iron deposition in MS. ${ }^{40}$

The current understanding of MS opens a view far beyond the single lesion. The hypothesis of CCSVI therefore ultimately fails in attempting to explain MS solely on the basis of perivenous MS lesions. This hypothesis cannot account for the sea of neurodegeneration surrounding the lesion; nor can it explain the vast heterogeneity of MS.

\section{Limitations}

One limitation of this study is a relatively small sample size. Small effects may be missed unless larger studies are performed. However, an effect of the magnitude claimed by Zamboni et al ${ }^{5}$ can be ruled out with sufficient certainty by the present findings. Second, the question as to whether or not abnormalities of the major venous conductors may be over-represented in MS patients was beyond the aim and scope of this study. Evidence pertinent to this issue will only become available from further independent venographic or contrast-enhanced sonographic investigations.

\section{Conclusion}

This triple-blinded controlled study does not support insufficient extra- and intracranial venous flow in MS. Together with two other recent studies, ${ }^{21} 22$ this constitutes compelling evidence against a significant contribution of CCSVI to the pathogenesis of MS. As interventional procedures such as transluminal angioplasty are derived from the non-confirmed CCSVI concept ${ }^{7}$ and can result in serious adverse events, ${ }^{13}$ we strongly discourage the use of these procedures on the grounds of the present evidence.

\section{Competing interests None}

Patient consent Obtained.

Ethics approval Ethics approval was provided by the Ethics Committee of the Medical Faculty, Goethe-University of Frankfurt, Frankfurt am Main, Germany.

Provenance and peer review Not commissioned; externally peer reviewed.

\section{REFERENCES}

1. Charcot JM. Histologie de la sclerose en plaque. Gaz Hosp Paris 1868;141:554-8.

2. Lassmann H. Axonal and neuronal pathology in multiple sclerosis: what have we learnt from animal models. Exp Neurol 2010;225:2-8.

3. Meinl E, Derfuss T, Krumbholz M, et al. Humoral autoimmunity in multiple sclerosis. J Neurol Sci Published Online First: 2 September 2010. doi:10.1016/j. jns.2010.08.009.

4. Compston A, Coles A. Multiple sclerosis. Lancet 2008:372:1502-17.

5. Zamboni $\mathbf{P}$, Galeotti R, Menegatti $\mathrm{E}$, et al. Chronic cerebrospinal venous insufficiency in patients with multiple sclerosis. J Neurol Neurosurg Psychiatry 2009;80:392-9.
6. Singh AV, Zamboni P. Anomalous venous blood flow and iron deposition in multiple sclerosis. J Cereb Blood Flow Metab 2009;29:1867-78.

7. Zamboni P, Galeotti R, Menegatti E, et al. A prospective open-label study of endovascular treatment of chronic cerebrospinal venous insufficiency. J Vasc Surg 2009;50:1348-58, e1-3

8. Bergan JJ, Schmid-Schonbein GW, Smith PD, et al. Chronic venous disease. N Engl J Med 2006;355:488-98.

9. Leriche H, Aubin ML, Aboulker J. Cavo-spinal phlebography in myelopathies. Stenoses of internal jugular and azygos veins, venous compressions and thromboses (In French). Acta Radiol Supp/ 1976;347:415-17

10. Schelling F. Damaging venous reflux into the skull or spine: relevance to multiple sclerosis. Med Hypotheses 1986:21:141-8.

11. Ogleznev KO, Tsuladze II. Diagnosis of venous circulation disorders in the cervical portion of the spine and spinal cord using selective phlebography (In Russian). Vestn Rentgenol Radiol 1993:46-9.

12. Talbert DG. Raised venous pressure as a factor in multiple sclerosis. Med Hypotheses 2008:70:1112-17.

13. None. Experimental multiple sclerosis vascular shunting procedure halted at Stanford. Ann Neurol 2010;67:A13-15

14. Barkhof F, Filippi M, Miller DH, et al. Comparison of MRI criteria at first presentation to predict conversion to clinically definite multiple sclerosis. Brain 1997;120:2059-69.

15. Polman CH, Reingold SC, Edan G, et al. Diagnostic criteria for multiple sclerosis: 2005 revisions to the 'McDonald Criteria.' Ann Neurol 2005:58:840-6.

16. Stolz E, Kaps M, Kern A, et al. Transcranial color-coded duplex sonography of intracranial veins and sinuses in adults. Reference data from 130 volunteers. Stroke 1999:30:1070-5

17. Schaller B. Physiology of cerebral venous blood flow: from experimental data in animals to normal function in humans. Brain Res Brain Res Rev 2004:46:243-60.

18. Valdueza JM, von Munster T, Hoffman 0, et al. Postural dependency of the cerebra venous outflow. Lancet 2000;355:200-1.

19. Gisolf $\mathbf{J}$, van Lieshout $\mathrm{J} J$, van Heusden $\mathrm{K}$, et al. Human cerebral venous outflow pathway depends on posture and central venous pressure. J Physiol 2004;560:317-27.

20. Schreiber SJ, Lurtzing F, Gotze R, et al. Extrajugular pathways of human cerebral venous blood drainage assessed by duplex ultrasound. J App/ Physio/ 2003;94:1802-5

21. Doepp F, Paul F, Valdueza JM, et al. No cerebrocervical venous congestion in patients with multiple sclerosis. Ann Neurol 2010;68:173-83.

22. Sundstrom P, Wahlin A, Ambarki K, et al. Venous and cerebrospinal fluid flow in multiple sclerosis: a case-control study. Ann Neurol 2010;68:255-9.

23. Nedelmann M, Eicke BM, Dieterich M. Functional and morphological criteria of internal jugular valve insufficiency as assessed by ultrasound. J Neuroimaging 2005; 15:70-5.

24. Nedelmann M, Kaps M, Mueller-Forell W. Venous obstruction and jugular valve insufficiency in idiopathic intracranial hypertension. J Neurol 2009;256:964-9.

25. Schreiber SJ, Doepp F, Klingebiel R, et al. Internal jugular vein valve incompetence and intracranial venous anatomy in transient global amnesia. J Neurol Neurosurg Psychiatry 2005;76:509-13

25a. Zamboni P, Menegatti E, Galeotti R, et al. The value of cerebral Doppler venous haemodynamics in the assessment of multiple sclerosis. J Neurol Sci 2009;282:21-7

26. van Bemmelen PS, Bedford G, Beach $\mathrm{K}$, et al. Ouantitative segmental evaluation of venous valvular reflux with duplex ultrasound scanning. J Vasc Surg 1989;10:425-31.

27. Sarin S, Sommerville K, Farrah J, et al. Duplex ultrasonography for assessment of venous valvular function of the lower limb. Br J Surg 1994:81:1591-5.

28. Lagattolla NR, Donald A, Lockhart $S$, et al. Retrograde flow in the deep veins of subjects with normal venous function. Br J Surg 1997:84:36-9.

29. Lichtenstein D, Saifi R, Augarde R, et al. The Internal jugular veins are asymmetric Usefulness of ultrasound before catheterization. Intensive Care Med 2001;27:301-5.

30. Doepp F, Schreiber SJ, von Munster T, et al. How does the blood leave the brain? A systematic ultrasound analysis of cerebral venous drainage patterns. Neuroradiology 2004:46:565-70.

31. San Millan Ruiz D, Gailloud P, Rufenacht DA, et al. The craniocervical venous system in relation to cerebral venous drainage. Am J Neuroradiol 2002;23:1500-8.

32. Gius JA, Grier DH. Venous adaptation following bilateral radical neck dissection with excision of the jugular veins. Surgery 1950;28:305-21.

33. Eckenhoff JE. The physiologic significance of the vertebral venous plexus. Surg Gynecol Obstet 1970;131:72-8.

34. Clemens HJ. Die Venensysteme der menschlichen Wirbelsäule. Morphologie und funktionelle Bedeutung. Berlin: Walter de Gruyter \& Co, 1961

35. Batson OV. Anatomical problems concerned in the study of cerebral blood flow. Fed Proc 1944;3:139-44

36. De Simone R, Ranieri A, Bonavita V. Advancement in idiopathic intracranial hypertension pathogenesis: focus on sinus venous stenosis. Neurol Sci 2010;31 (Suppl 1):S33-9.

37. Sugarbaker ED, Wiley HM. Intracranial-pressure studies incident to resection of the internal jugular veins. Cancer 1951:4:242-50.

38. Williams BJ, Skinner HJ, Maria BL. Increased intracranial pressure in a case of pediatric multiple sclerosis. J Child Neurol 2008;23:699-702.

39. Bechmann I, Galea I, Perry VH. What is the blood-brain barrier (not)? Trends Immunol 2007;28:5-11.

40. Worthington V, Killestein J, Eikelenboom MJ, et al. Normal CSF ferritin levels in MS suggest against etiologic role of chronic venous insufficiency. Neurology 2010;75:1617-22. 